\title{
Incidencia del estreptococo en la producción del sindrome nefrítico agudo en la infancia
}

\author{
DRS. FEDERICO PUGA *, EDUARDO DONOSO *, GASTON ZILLERUELO *, RICARDO SORENSEN *, y \\ SRA. HILDA KUMZAGK *.
}

El Sindrome Nefrítico Agudo en la infancia tiene muy diversas etiologías (1-2-3-4-5-6-8-9 15-16), pero la mayoría de ellos son consecuencia de infecciones por Estreptococo del Grupo A con sus tipos nefritógenos. La importancia del diagnóstico etiológico del Sindrome de Glomérulonefritis Aguda reside en que aquellos que son consecuencias de infecciones estreptocócicas ticnen una recuperación entre un 91 al 100\% (14-17), en un plazo bastante corto según las técnicas que se usen para considerar la mejoría. Esta garantía favorable, sin embargo requiere que el diagnóstico etiológico de la estreptococia sea bien demostrado al comienzo de la enfermcdad.

Clínicamente la infección estreptocócica puede sospecharse en un $70 \%(10-12)$ de los casos, pero habitualmente los niños llegan a consultar por su nefropatía varios días después, cuando aquella va en regresión. (19-20). El cultivo de la secreción faríngea tampoco es de un rendimiento alto, habiéndose encontrado alrededor de un $38 \%$ ( 11 ) de cultivos positivos en pacientes con Nefritis, resultado que todavía es menos fayorable que si el paciente ha sido tratado previamente con antibióticos.

Dada la importancia que tiene para el pronóstico el diagnóstico etiológico es que se han usado las determinaciones séricas de la antiestreptolisina 0 (13-18) y otras que nos permiten confirmar la infección previa por el estreptococo; la determinación de las antiestreptolisinas 0 (ASO) es la más difundida.

Ultimamente el factor 3 del complemento (C3) se ha agregado como elemento útil en el diagnóstico de la etiología de la Glomérulonefritis Aguda ya que en Pediatría son pocas las Nefropatías que cursan con un $\mathrm{C} 3$ bajo.

Solamente el lupus y escasas Glomérulonefri-

* Hospital Lujs Calvo Mackenna. tis crónicas (21) entrarían en el diagnóstico diferencial y sus características clínicas además de su escasa frecuencia hacen que el C3 bajo signifique en la infancia una glomérulonefritis aguda estreptocócica. Se analiza la experiencia de los años 1971 hasta Julio de 1972 en 81 pacientes con Sindrome de Glomérulonefritis Aguda (G. N. A.) en los cuales se hizo la determinación de antiestreptolisina 0 y determinación de complemento con el objeto de apreciar la incidencia de la estreptococia en la etiología de la Glomérulonefritis Aguda en nuestro medio.

Material $\chi$ MÉTodo. Se analizan 81 niños con glomérulonefritis aguda hospitalizados en el Hospital Luis Calvo Mackenna en los años 1971 a Julio de 1972. Hubo $70 \%$ de varones y $30 \%$ de mujeres cuyas edades fluctúan entre 3 y 14 años.

En 57 casos se investiga con cultivo de secreción faríngea la existencia de estreptococo Grupo A. En todos se hizo, por lo menos, una determinación de antiestreptolisina 0 antes de Ios 10 días de iniciada la enfermedad renal y en 26 pacientes, una segunda determinación entre la $3^{\mathrm{a}}$ y 5 a semana de evolución de la enfermedad renal. Se considera como un título de antiestreptolisina elevado cuando éste es superior a 333 unidades Todd. Según la anamnesis, el cuadro clínico, exámenes de laboratorio, evolución y títulos de antiestreptolisina bemos dividido nuestros pacientes en tres grupos:

1.- Cuadro clínico completo de gloménilonefritis aguda, antecedentes de infección faríngea o de la piel. Evolución favorable en un plazo inferior a 12 meses, y título de antiestreptolisina 0 superior a 333 unidades Todd. A este grupo lo consideramos como nefritis de origen post-estreptocócica. 
2.-- Igual al anterior, pero con título de antiestreptolisina normal. Denominamos a este grupo, nefritis post-estreptocócica probable.

3.- Cuadro clínico de Glomérulonefritis Aguda sin antecedentes de Infección y título de antiestreptolisina normal. Denominamos a estc grupo nefritis de etiología desconocida.

En 39 de estos mismos pacientes se deterninó el factor 3 del complemento (C3) precozmente y se busca su importancia en el diagnóstico etiológico que será analizado. Cifra normal es 150 más o menos.

Resultado y COMENTario. El pronóstico de la glomérulonefritis aguda en el niño está condicionado estrechamente a su etiología. Si esta cs debica a una infección estreptocócica, habitualmente del arbol respiratorio alto o de la piel, el pronóstico de recuperación total en un plazo inferior a 12 meses es de alrededor de un $97 \%$ (14-17-19), becho que no ocurre con esta frecuencia en el total de los sindromes de glomérulonefritis aguda de la infancia. De este hecho deriva la importancia de pesquisar con distintos medios la existencia de una infección estreptocócica previa a la nefropatía.

El examen físico de estreptococia junto con la anamnesis nos permite precisar esta etiología en más o menos el $70 \%$ de los pacientes, pero los pacientes llegan a consultar después de algunos días de pasado el cuadro infeccioso faríngeo o de la piel y aún, muchos de ellos han recibido terapias antibióticas.

El hallazgo del estreptococo Grupo $A$ en la faringe del enfermo nefropata es bajo (12\%) y en esta experiencia, el hecho de encontrario en la faringe no permite concluir que la nefropatía sea debida a ese estreptococo (19).

Es por estas razones que se ha usado la determinación de la antiestreptolisina 0 del suero y el factor 3 del complemento para determinar la existencia de una infección estreptocócica previa, la que tomada precozmente, al comienzo de la nefropatía, nos darán títulos de estreptolisina elevado y valores de complemento bajo, si la causa es el estreptococo.

Sin embargo el hallazgo de antiestreptolisina elevados y valores de complemento bajo, si la caupara concluir que la etiología es el estreptococo siendo de mayor utilidad la determinación de una curva de antiestreptolisina. La curva en ascenso o descenso en la antiestreptolisina seria positiva de la etiología estreptocócica, siendo importante destacar que es indispensable la determinación precoz de la antiestreptolisina 0 y el factor 3 al comienzo de la Nefropatía para que esta curva tenga algún valor. Si no hemos hecho el diagnóstico etiológico en este plazo relativamente corto de 4 a 6 semanas, posteriormente no tendremos ninguna manera de confirmarlo.

De acuerdo con el análisis anamnéstico el cuadro clínico y la determinación de antiestreptolisina hemos separado nuestra casuística en 3 grupos: Tabla $\mathrm{I}$.

$$
\text { T A B L A I }
$$

DISTRIRUCION SEGUN ETIOLOGIA

81 pacientes con G.N.A.

\begin{tabular}{|c|c|c|c|c|c|c|c|}
\hline Grup & $10 N^{\circ}$ & $\begin{array}{l}\text { Ant. } \\
\text { infec. }\end{array}$ & $\begin{array}{l}\text { Cuadro } \\
\text { clínico }\end{array}$ & A.S. $O$. & $\begin{array}{l}C^{\prime} \\
\text { bajo } \\
\%\end{array}$ & Efiol. & $\%$ \\
\hline I & 53 & (Sí) & G.N.A. & Alta & 84 & Estrep. & 65 \\
\hline II & 21 & $(S i)$ & $"$ & Norm. & 73 & $\begin{array}{l}\text { Estrep. } \\
\text { probable. }\end{array}$ & 26 \\
\hline III & 7 & (No) & $"$ & Norm. & 0 & $\begin{array}{l}\text { Descono- } \\
\text { cida }\end{array}$ & 9 \\
\hline
\end{tabular}

1 - 53 niños $(65 \%)$ con diagnóstico de glomérulonefritis aguda post-estreptocócica confirmada.

2.- 21 casos $(26 \%)$ de glomérulonefritis probablemente estreptocócica.

3.- 7 casos $(9 \%)$ con diagnóstico de glomérulonefritis aguda de etiología desconocida.

De acuerdo con estos resultados, el $91 \%$ de los sindromes de glomérulonefritis aguda observados durante los años 71 y hasta Julio del 72 son debidos a una infección estreptocócica entre las cuales en un $65 \%$ ésta es confirmada y en un $26 \%$ ella es probable. Quedan sin embargo, 7 pacientes $(9 \%)$ cuya etiología estreptocócica no está confirmada y tampoco hay datos anamnésticos que nos premitan sospecharla. De los 21 pacientes con glomérulonefritis aguda probablemente estreptocócica el $82 \%$ tuvieron un $\mathrm{C}_{3}$ bajo demostrando en esta forma que, a pesar de la negatividad de la antiestreptolisina de etiología más probable de esos pacientes es la etiología estreptocócica. Por otro lado en el Grupo 3 de pacientes cuya etiología es desconocida de los 7 del total se practicaron en 5 de ellos la determinación de $C_{3}$ siendo en todos ellos negativo, con lo que se acuerda la posibilidad de que la etiología de este grupo no sea estreptocócica.

Varias razones pueden aducirse para explicar la existencia de estas antiestreptolisinas 0 en el Grupo 2 con complemento bajo.

En primer lugar la puerta de entrada es evidente que influye en la elevación de las antiestrep- 
tolisinas siendo esta alza muy superior cuando la puerta de entrada es faringea y mucho menor o normal cuando la puerta de entrada es a través de infecciones de la piel. Es así, como las antiestreptolisinas 0 estuvieron elevadas en el $77 \%$ de las infecciones estreptocócicas de puerta de entrada faringea, solamente en el $50 \%$ de aquellas con puerta de entrada de la piel. Tabla II. Por otro lado es conocido que hay gérmenes malos productores de antígenos. $Y$ por último, los plazos a contar de la infección en que se toman las muestras para antiestreptolisinas. Si este plazo es muy precoz muy al comienzo de la nefropatía y ésta ha estado muy cerca de la infección faringea, pueđe el título ser negativo como ocurrió en uno de nuestros pacientes y posteriormente subir. $O$ también cuando la determinación se practica des. pués de la 6a semana cuando los títulos pueden haber regxesado a niveles normales. De alli la necesidad que las muestras seriadas nos serán útil solamente si una es precoz y la otra de la $3^{\text {a }}$ a la 5 a semana de enfermedad.
TABLA I I

PUERTA DE ENTRADA ESTREPTOCOCO

$Y$ TITULO DE ASO

81 pacientes con G. N. A.

\begin{tabular}{lcrr}
\hline & $N^{o}$ & ASO alta & $\%$ \\
\hline Faringe & 48 & 37 & 77 \\
Piel & 10 & 5 & 50 \\
Sin ant. & 23 & 11 & 48 \\
\hline
\end{tabular}

En lo que se refiere a la determinación del complemento $\left(\mathrm{C}_{3}\right)$, en pediatría existen muy pocas patológicas nefrológicas que aumenten el consumo del complemento. Solamente conocemos la glomérulonefritis aguda post-estreptocócica, el lupus eritemetoso diseminado agudo y un reducido número de glomérulonefritis crónicas que evolucionan permanentemente con un complemento bajo.

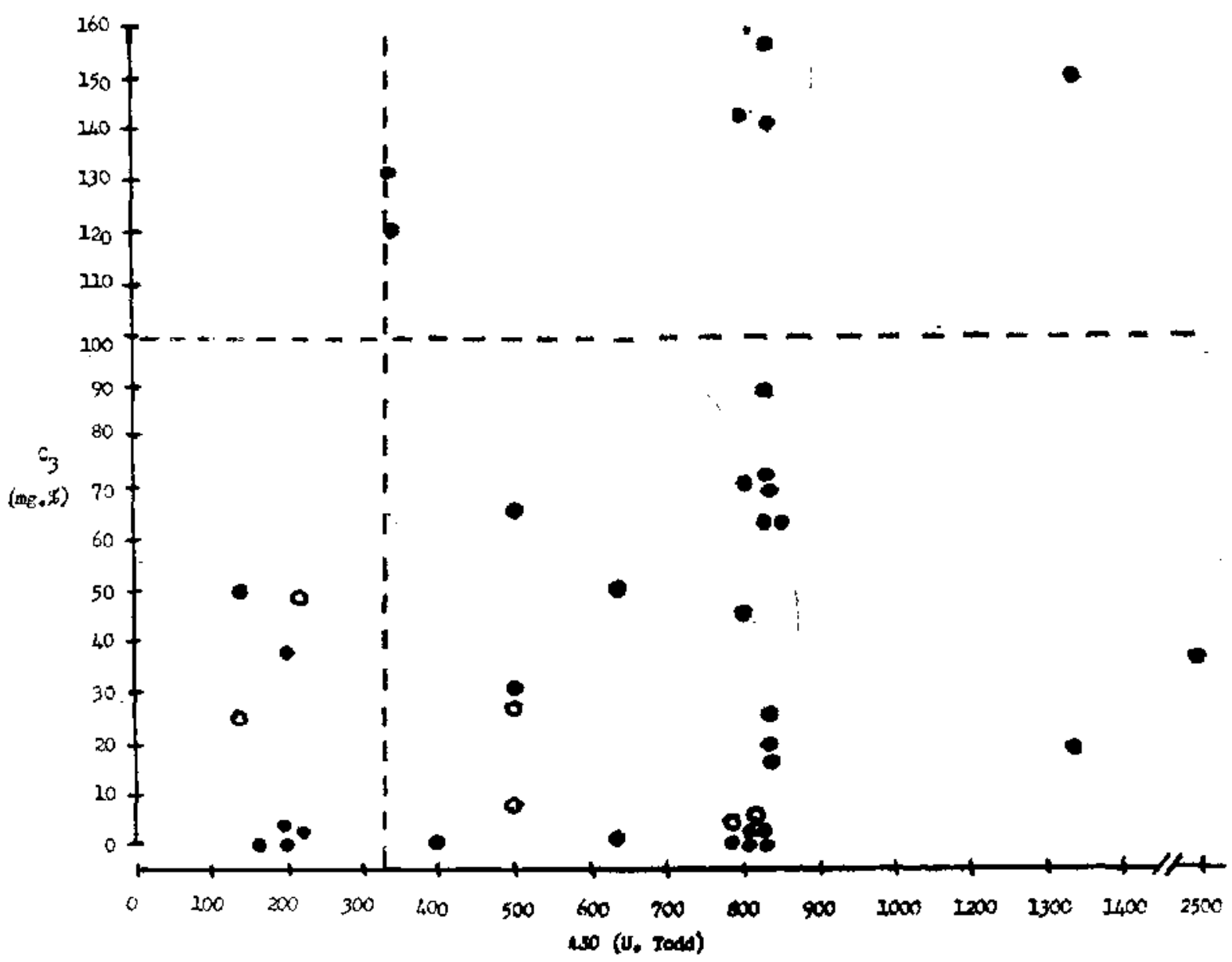

VALORES DE ANTIESTREPTOLINA “O” Y COMPLEMENTO $\left(C_{3}\right)$ EN 40 G. N. A. 
Es por esta razón que la reducción del complemento la consideramos como otro elemento más para la pesquisa etiologica de la glomérulonefritis aguda ya que es conocido que en pediatría el lupus es extraordinariamente infrecuente y cxisten muy escasas publicaciones referidas a aquellas Glomérulonefritis crónicas con complemento bajo. Vale la pena concluir que ante la existencia de un complemento bajo la primera sospecha debe ser una aefritis de etiología estreptocócica, ya que las otras dos causas que reducen el complemento tienen manifestaciones clínicas evidentes que nos permiten fácilmente diferenciarla de la glomérulonefritis aguda post-estreptocócica.

Si con este criterio sumamos los pacientes que tienen título de antiestreptolisina elevado con aquellos que tienen el complemento bajo encontramos un total de 66 pacientes lo que nos da un $81 \%$ de glomérulonefritis aguda en este grupo estudiado que puede ser atribuible al estreptococo Beta hemolítico de los Grupos A nefritógenos. Todo este esfuerzo que debe realizarse al comienzo de la nefropatía ticne un alto valor en lo que se refiere al pronóstico ya que si la netropatía es debida a una infección estreptocócica, las probabilidades de recuperación son muy cercanas al $100 \%$, cosa que no ocurre si tomamos el total de casos con un sindrome de glomérulonefritis aguda cuya etiología puede ser desconocida. En la figura No 1 , puede observarse que en el sindrome de glomérulonefritis aguda elevadas corresponden con $\mathrm{C}_{3}$ bajo certificando un mecanismo antïgeno-anticuerpo de cuya etiología nos habla la ASO.

Existe un pequeño grupo con ASO elevada y complemento normal, lo que podría deberse a que este último hubiera normalizado precozmente y otro grupo con ASO normal y $\mathrm{C}_{3}$ bajo, cuyas posibles explicaciones ya han sido mencionadas.

Nos parece que con ASO elevada o $C_{3}$ bajo podemos postular la etiología estreptocócica de la glonérulonefritis aguda ya que las otras posibilidades son raras y existen elementos para hacer el djagnóstico diferencial.

Conclusión. Con bastante aproximación podemos concluir que el estreptococo es la causa del Sindrome de Glomérulonefritis Aguda en los pacientes atendidos entre los años 71 y $72 \mathrm{cn}$ aproximadamente un $61 \%$. Sin embargo, esta frecuencia puede sufrir variaciones de año en año ya que el año 72 nosotros contamos con una epidemia de Escarlatina que sabemos causante de Glomérulonefritis Aguda. Vale la pena destacar esta frecuencia ya que el tratamiento a base de penicilina es elemental y además debemos insistir en que el tratamiento debe hacerse también a los contactos del paciente, ya que también ellos están expuestos a hacer una glomérulonefritis aguda aunque ésta sea inaparente, en especial aquellos contactos de edad infantil.
Otro valor de esa pesquisa etiológica es el excelente pronóstico que tienen las glomérulonefritis aguda cuando se logra demostrar que la etiología es estreptocócica.

No tenemos explicación de la etiología del restante $19 \%$ de nuestros pacientes ya que no tenemos estudio histológico en la mayoría de ellos.

\section{Resumen}

Se presentan los resultados de las pesquisas de la etiología estreptocócica en 18 pacientes con glomérulonefritis aguda encontrando esta como causa positiva a traves de la determinación de antiestreptolisina en un $65 \%$ de los pacientes, como causa probable en un $26 \%$ y como de etiología desconocida un $9 \%$. Si a la determinación de antiestreptolisina las complementamos con la determinación de $C_{3}$ encontramos que presentan titulo de antiestreptotisina alto y complemento bajo 66 pacientes (81\%) cuya etiología más probable es la estreptocócica.

Se destaca la importancia de la determinación precoz de antiestreptolisina o y de complemento al comienzo de la enfermedad renal.

\section{SUMMARY}

Antistreptolysin 0 is used in search of the etiology of 81 patients with A. G. N. In $65 \%$ the titer is high with a history of previous throat infection, artifying the streptococal cause. In $26 \%$ the history of previous infectionis positive but A. S. O. is within normal titers and in $9 \%$ the history nor the A. S. O. titers are positive.

Complement (C3) was also determined and $66 \mathrm{pa}$ tients had A. S. O. with high titers and or low C3, mearing that $81 \%$ of these patients could he considered as post streptocosal nephritis.

Analysis of faulse negative A. S. O. and C3 are considered.

\section{BIBLIOGRAFÍA}

1.-Bates R. C., Jenning R. B., y Earle D. F. Acute nephritis unrelated to group $A$ hemolitic strep. infections. Amer. J. Med. 23: 510, 1957.

2... Earle D. P., y Jening D. P. Post strep. glomerulonephritis. J. Clin. Invest. 40: 1525, 1961.

3.-Wannamaker L. W., Pierce H. C. Family outbreak of acute nephritis asociated with type 49 strep infections. Lancet 81: 561, 1961.

4.-Wertheim A. R., Lyttle J. D., Loeb E. N., Earle D. $P$., Seegal B. C., y Seegal D. The associations of type especific hemolytic. Strep with acute nephritis. J. Clinic Invest. 32: 319, 1963. 
5.- Rammel Kampof C. H, Microbiologyc aspects of Glomerulonephritis. J. Chronic Dis. 5: 28, 1967.

6.- Kleinman H. Epidemic acule Glomerulonephritis at Red lake. Mjnnesota Med. 37: 479, 1954.

7.- Futaher P. H. Glomerulonephritis following infections of the skin. Archi Intern. . Med. 65; 1192, 1940.

8.-Blemberg R. W., Feldman D. B. Observations or acute Glomerulonephritis associated with impetigo. J. Pediatr. 60: 677, 1962.

9.-. Simón N. S., Patter E. V., Siegel A. C., Mc Aninch J, Poon-King T., Herman L., y Earse D. P. Epidemic nephritis in Trinidad Proc. Cent. Societed Clinic Research, 38: 73, 1965.

10.-Homkman L. A., Massel B. F. Guidelines for diagnosic of strep infections. Pediat. 48: 573, 1971 .

11.-Puga F. Nefrología. Ed. Andrés Bello, 1966.

12.- Wannamaker $L$, W. Differences between Streptococo infections of the clinvat and of the skin. New Eng. J. Med. 282: 23, 1970.

13.--Id. 282: 78, 1970.
14.-Greifer I. Clinics, pathology and natural history of acute Glomerulonephritis Pag. 167. Acute Glomerulonephritis. Ed. Jack Metkoff. Little Brown, 1967.

15.-Yuccoglu A. M., Bercovich S. Acute Glomerulonephritis associated with chikenpox and Echo virux, Type 9 infections. Glomerulonephritis. Ed. Metkoff.

16.-Virus y Riñón. Revista Chilena de Pediatría. 38; $92,1967$.

17.-F. Puga. Glomerulonefritis Aguda, Nefrología. Pag. 84. Ed. Andrés Bello, 1966.

18.- Antiestreptolisinas e's nefropatias. Revista Chilena de Pediatría. 35: 229, 1964.

19.- Mc Crory W. W. y Shibuya M. Post streptococo Glomerulonephritis in children. Ped. Clinic. 11: $633,1964$.

20.- Puga F, Minica I., Medina E., Domic C. Algunos aspectos de la Glonźruloncfritis del niño, Revista Cbilena de Pediatría. 37: 41, 1966.

21.-Glomerulonephritis estreptocócica hipo y monocomplementemica. Pediatric Research. 6: 330, 70, 1972. 